\title{
The Immunologic Role of Gut Microbiota in Patients with Chronic HBV Infection
}

\author{
Ruilin Yang $\mathbb{D},{ }^{1}$ Yao Xu, ${ }^{1}$ Zhifeng Dai, ${ }^{1}$ Xuhong Lin $\mathbb{D}^{1},{ }^{1}$ and Huichao Wang ${ }^{2}$ \\ ${ }^{1}$ Department of Clinical Laboratory, Translational Medicine Center, Huaihe Hospital Affiliated to Henan University, Kaifeng, \\ Henan 475000, China \\ ${ }^{2}$ Department of Nephrology, First Affiliated Hospital of Henan University, Kaifeng, Henan 475000, China
}

Correspondence should be addressed to Xuhong Lin; 8007261xh@tongji.edu.cn

Received 5 January 2018; Revised 15 May 2018; Accepted 29 May 2018; Published 25 July 2018

Academic Editor: Lucia Conti

Copyright (c) 2018 Ruilin Yang et al. This is an open access article distributed under the Creative Commons Attribution License, which permits unrestricted use, distribution, and reproduction in any medium, provided the original work is properly cited.

Hepatitis B can cause acute or chronic liver damage due to hepatitis B virus (HBV) infection. Cirrhosis or hepatocellular carcinoma (HCC) caused by chronic HBV infection often leads to increased mortality. However, the gut and liver have the same embryonic origin; therefore, a close relationship must exist in terms of anatomy and function, and the gut microbiota plays an important role in host metabolic and immune modulation. It is believed that structural changes in the gut microbiota, bacterial translocation, and the resulting immune injury may affect the occurrence and development of liver inflammation caused by chronic HBV infection based on the in-depth cognition of the concept of the "gut-liver axis" and the progress in intestinal microecology. This review aims to summarize and discuss the immunologic role of the gut microbiota in chronic HBV infection.

\section{Introduction}

Chronic hepatitis B virus (HBV) infection is a global problem that threatens human health. Chronic HBV infection can easily lead to some severe complications, such as liver failure, cirrhosis, and even hepatocellular carcinoma (HCC), which can be life-threatening if not treated in a timely and normative manner. According to the latest report from the World Health Organization (WHO), there are approximately 257 million people with chronic HBV infection worldwide, and of these cases, 887,000 people die due to complications caused by chronic HBV infection [1]. Although the application of the hepatitis $B$ vaccine and antiviral drugs has caused the rate of HBV infection to decrease yearly, chronic HBV infection still remains a heavy economic burden and health threat for many families $[2,3]$.

Researchers have persisted in finding better ways to control this disease. In recent years, studies on intestinal flora have provided new targets for the prevention and treatment of chronic HBV infection. Studies have demonstrated that changes in the gut microbiota play important roles in inducing and promoting the development of liver diseases, and the diversity of the gut microbiota has been associated with alcoholic liver disease, autoimmune liver disease, chronic hepatitis B (CHB), cirrhosis and HCC; moreover, changes in the intestinal flora are dependent on the various pathogeneses that are present [4-8]. Furthermore, scholars from Taiwan have indicated that the gut microbiota may determine acute hepatitis B and CHB attacks. Specifically, the composition of the gut microbiota affects the host immune response to $\mathrm{HBV}$, and the infection can easily transform into a chronic infection when the intestinal flora is in an abnormal state [9]. Accordingly, the fact that immune injuries caused by structural changes in the gut microbiota have a significant influence on the development of chronic HBV infection cannot be ignored.

\section{Chronic HBV Infection and Innate Immunity}

Adults generally experience acute onset after infection with HBV. However, most people are able to clear the virus via a healthy immune system, and only a few individuals with impaired immunity or other liver diseases progress to chronic HBV infection. Most chronic HBV infections occur in infants and young children due to their immature immune systems and unstable intestinal flora $[10,11]$. Therefore, the 
occurrence and development of chronic HBV infection not only depend on the viral load, virulence, and invasion path of the HBV but also are associated with the immune function and intestinal flora of the host. It is currently believed that the cause of liver injury is not HBV replication in liver cells but rather the immune response caused by the HBV. The immune response is considered a key factor in the development of CHB. Various immune cells and cytokines not only are involved in the initiation and regulation of the immune response but also can activate downstream signaling pathways that directly or indirectly inhibit HBV replication. It can be concluded that the host immune response caused by $\mathrm{HBV}$ infection exerts a significant influence on the prognosis of hepatitis B and the treatment effect of antiviral drugs [12]. The immune response against HBV mainly includes innate immunity and adaptive immunity; however, the effective innate immune response not only can eliminate the virus directly but also can exert a significant influence on $\mathrm{HBV}$ specific immunity [13].

Toll-like receptors (TLRs) are important protein molecules involved in innate immunity. Acting as a bridge connecting nonspecific immunity and specific immunity, they recognize molecules with conserved structures derived from microorganisms. After the breach of the body's physical barriers, such as the skin or mucous membranes by microorganisms, TLRs identify molecules and become activated, leading to immune responses [14]. TLRs are expressed in multiple liver immune cells, including plasmacytoid dendritic cells ( $\mathrm{pDCs}$ ), monocytes, macrophages, Kupffer cells, hepatic stellate cells, and liver cells [12]. Experimental studies have indicated that the activation of cell signaling pathways by TLRs and the release of antiviral cytokines are the main mechanisms by which HBV replication in liver cells is suppressed. When the host is infected by HBV, the virus is identified by the relevant TLRs, and the mechanisms of antiviral regulation are activated. These mechanisms include TLR recognition, the release of interferon (IFN), activation of NK and NKT cells, and the production of proinflammatory cytokines [14]. For example, the activation of the HBV-TLR3 signaling pathway causes the production of IFN- $\alpha / \beta$, and TLR2/TLR4 can initiate the MAPK and PI3K/Akt signaling pathways [15]. However, another research has demonstrated that $\mathrm{HBV}$ can reduce the expression of TLR2 in liver Kupffer cells and mononuclear cells in patients with chronic HBV infection and can also attenuate intracellular signaling pathways to enable the evasion of the immune response [16]. A recent study also found that the HBV DNA viral load is negatively correlated with TLR7 expression in biopsy samples, which indicates that expression of TLR7 on liver cells can be inhibited by HBV [17]. Obviously, the TLR-mediated signaling pathways induce immune responses to $\mathrm{HBV}$ infection, but the $\mathrm{HBV}$ virus itself downregulates the expression of TLRs on the immune cells. The latter mechanism may be one of the main causes of progression to chronic HBV infection in patients. Studies on the gut-liver axis have revealed that the intestine and liver have the same embryonic origin and are linked by the portal venous system. The intestinal tract has been proposed to exert a regulatory effect on the development of chronic HBV infection. The intestinal flora is an important mediator of the interaction between the intestine and the liver and the portal vein functions as a bridge connecting these structures [8].

\section{Gut Microbiota}

As the name suggests, the gut microbiota includes microorganisms that have colonized and coexist in the human intestinal tract. Due to host genetic, diet, and environmental factors and use of antibiotics, certain differences exist in the components of the human intestine among individuals, but the human body will gradually establish a stable intestinal flora structure and regulate and maintain the body's health under the influence of these factors [18-20]. Tens of thousands of microbes live in the intestinal tracts of normal adults, and most of these microbes are located in the colon. The number of bacteria located in the colon of the human body is estimated to be approximately $3.8 \times 10^{13}$ according to the identified bacterial content and colon volume. There are approximately 40,000 types of microbes in the gut and can be divided into two main categories, that is, Bacteroides and the thick-walled bacteria. The most common bacteria are Bifidobacteria, Lactobacillus, Clostridium, and Streptococcus [21]. These intestinal bacteria are considered beneficial, and their roles in metabolism, immunity, and nutrition can prevent invasion of the host by disease-causing pathogens [22]. Thus, the intestinal immune function cannot be neglected. Data of experiments showed that imbalances of the intestinal flora easily lead to disorders of the intestinal immune system and cause a variety of diseases such as irritable bowel syndrome (IBS), inflammatory bowel disease (IBD), autoimmune liver disease, and $\mathrm{CHB}$ [23-26]. Moreover, the gut microbiota is involved in nonalcoholic fatty liver disease (NAFLD) and associated with the progression of NAFLD to nonalcoholic steatohepatitis, cirrhosis, or HCC $[19,27]$.

\section{Immune Function of Gut Microbiota in the Development of Chronic HBV Infection}

In recent years, many scholars, especially domestic scholars, have studied the relationship between gut microbiota and chronic HBV infection because of the high proportion of $\mathrm{HBV}$-infected people worldwide. In 2006, Xing et al. found that liver ischemia and reperfusion can reduce the numbers of intestinal Bifidobacteria and Lactobacillus and increase the numbers of Enterobacteriaceae and Enterococcus; these changes are associated with the loss of intestinal microvilli, the widening of the intestinal mucosal space and intestinal bacterial translocation [28]. Subsequently, the same changes were found in the intestinal flora of chronic HBV carriers, CHB patients, and hepatitis B-induced cirrhosis patients; that is, the structures and abundances of the bacterial groups were obviously different. Specifically, patients with CHB and cirrhosis exhibit dramatically decreased Bifidobacteria and Lactobacillus levels, while Enterococcus and Enterobacteriaceae levels are significantly increased compared to healthy people. The progression of liver diseases, particularly liver cirrhosis, is caused by bacterial products from the intestine $[29,30]$. Chou et al. found that HBV could not be detected 
after six weeks of HBV infection in adult mice without intestinal flora (treated by antibiotics), and $60 \%$ of adult mice with intestinal flora (no antibiotics) still exhibited HBV. These data imply that gut microbes play a critical role in immunity against HBV [9]. Wang et al. studied the intestinal flora of $\mathrm{CHB}$ patients and healthy people, and the data revealed that the Bacteroides level was decreased in CHB patients compared to healthy people based on sequencing the V3-V4 region of the $16 \mathrm{~S}$ rRNA gene of the intestinal flora. Additionally, these authors also discovered that the intestinal microflora structure of the CHB patients had changed compared to that before severe liver injury, which indicated that the structural changes in the intestinal flora played a potential pathogenic role in patients with chronic HBV infection [31]. In summary, an imbalance exists in the intestinal microbiota of patients with $\mathrm{CHB}$ and cirrhosis. Similarly, overgrowth of harmful bacteria in the intestinal tract leads to increased mucosal permeability, which causes harmful bacteria to travel through the portal vein into the liver and thus activate the liver's innate immune system. The structural changes in the intestinal microflora and the severity of liver disease are mutually causal, and to a certain extent, they affect the transformation process of $\mathrm{CHB}$ to liver fibrosis or liver failure [12]. Therefore, we suggest that, in chronic HBV infection, the injury to hepatocytes not only originates from the cellular immune response caused by HBV invasion but also is caused by the natural immune response elicited by pathogen-associated molecular patterns (PAMPs) produced by the intestinal microbes with structural disorders. TLRs are the main pattern recognition receptors in the natural immune system and play a crucial role in the immune response [12]. It is now known that the intestinal PAMPs associated with chronic HBV infection are mainly composed of lipopolysaccharide (LPS), unmethylated CpG DNA, bacterial cell wall components, and bacterial DNA/RNA. The related immune mechanisms are described below.

4.1. The LPS-TLR4 Pathway. LPS is the main component of the outer membrane of Gram-negative bacteria and is an endotoxin mainly released by Enterobacteriaceae. Research has demonstrated a high level of LPS in the blood of patients with chronic HBV liver failure, which indicates that LPS may be related to the severity of the disease [32]. Another study found that LPS in the intestinal tract can downregulate the expression of tight junction proteins ( $\mathrm{ZO}-1$ and closed protein), increase the permeability of the intestinal mucosa, and enter the blood flow through the portal venous system $[33,34]$. LPS can be recognized by TLR4, and TLR4 is mainly expressed in mononuclear macrophages [32]. Chou et al. [9] found that mice, regardless of age, subjected to TLR4 silencing are able to clear HBV and obviously produce antibodies in 8 weeks. In other words, TLR4-silenced mice are not immune to LPS but can clear HBV. Conversely, LPSinduced immune responses may contribute to the progression of chronic HBV infection. Researches showed that LPS binds to LPS-binding protein, and this combination can be identified by TLR4 on the surface of mononuclear macrophages. This recognition then stimulates $\mathrm{CD} 14^{+}$Kupffer cells, triggers the inflammatory cascade effect, activates the
NF- $\kappa$ B-related pathway, and produces inflammatory cytokines, such as tumor necrosis factor- $\alpha$ (TNF- $\alpha$ ), IL- 1 , and IL-6, and thus causes acute liver injury $[35,36]$. Simultaneously, this pathway also induces Kupffer cells to release immunosuppressive mediators, such as IL-10, which can inhibit the release of inflammatory mediators of mononuclear macrophages and HBV-specific immune responses, in turn inhibiting the efficient clearing of bacteria and HBV [37]. Additionally, hepatic stellate cells also express TLR4 and can release a large number of extracellular matrix proteins in a LPS-TLR4 pathway-dependent manner. These proteins are involved in the fibrotic process and may also be among the factors that cause chronic HBV infection to develop into liver fibrosis [38].

4.2. The Unmethylated CPG DNA-TLR9 Pathway. Unmethylated CpG DNA is an important immune adjuvant that can activate TLR9. TLR9 is mainly expressed on mononuclear cells, B cells, $\mathrm{CD}^{+} \mathrm{T}$ cells, pDCs, and Treg cells [39]. The CpG-TLR9 signaling pathway not only activates the innate immune response but also adjusts the adaptive immune response. The CpG-TLR9 signaling pathway plays an important role in the prevention and treatment of infectious diseases [40]. Researches have highlighted that CpG-TLR9 and MyD88 form compounds (MyD88, IRAK4, and TRAF6), and TRAF6 phosphorylates IRAK1 to trigger the NF- $\kappa \mathrm{B}$ and MAPK signaling pathways, which may activate DCs to express and secrete cytokines and chemokines to further promote the proliferation and differentiation of $\mathrm{B}$ and $\mathrm{T}$ cells, resulting in the secretion of proinflammatory cytokines and IFN [40,41]. However, different bacteria have different $\mathrm{CpG}$ DNA levels. In the intestinal flora of animals, unmethylated CpG DNAs are abundant in Lactobacillus casei, Lactobacillus plantarum, Lactobacillus rhamnosus, Bifidobacteria, Proteobacteria, and Bacteroidetes [40]. As mentioned above, the intestinal floras of chronic HBV-infected patients with aggravated conditions are highly maladjusted, and Lactobacillus and Bifidobacteria with richer unmethylated CpG DNA levels are greatly reduced, causing the weakening of the $\mathrm{CpG}$ DNA-TLR9 pathway, decreasing of the production of protective cytokines, especially IFN, and diminishing of the immune effect on HBV.

4.3. Other Pathways. Other components of intestinal bacteria can also be identified by liver immune cells, such as the following cell wall components: teichoic acid (TA), peptidoglycan (PGN), and specialized proteins (flagellin). TA and PGN are recognized by TLR2, and flagellin mainly activates TLR 5 $[42,43]$. TLR3 can combine with dsRNA in bacteria, and ssRNA can activate TLR7 and TLR8 receptors [44]. A series of protective immune responses depend on the MyD88TRIF pathway, which triggers the downstream signal and then initiates the activation of related immune cells to release proinflammatory cytokines [45]. Nevertheless, this process will also aggravate liver injury if the immune response persists for a long time or reaches an excessive intensity, which then results in the production of a large number of cytokines in the body [32]. 


\section{Summary and Prospects}

In conclusion, studies have confirmed that imbalances of the intestinal flora play an important role in promoting the development of chronic HBV infection. The PAMPs of intestinal bacteria are transferred into the liver through the portal vein and identified by TLRs in the immune cells, which causes a series of immune responses, the release of various cytokines (IL, TNF, and IFN), and further liver cell damage. Among these processes, TLRs play an essential role as an important link between the intestinal flora and the liver immune reaction.

Although antiviral drugs and IFN have significant efficacy in the treatment of chronic HBV infection, for various reasons, it seems inevitable that chronic $\mathrm{HBV}$ infection develops into liver cirrhosis, liver failure, or liver cancer. Currently, studies on the gut microbiota and its products have provided a new therapeutic target. An article proposed that the application of synthetic unmethylated CpG DNA is a promising measure for the treatment of infectious diseases [41]. Additionally, the physiological indexes (BMI) and serum metabolites of the patients with chronic HBV infection also correlated with the structure of the intestinal flora and liver fibrosis [31]. Certain physical exercises may actively regulate the intestinal floras of patients with chronic diseases [46].

In recent years, probiotics have been widely used in the treatment of intestinal diseases. Many studies have demonstrated that compound probiotics can improve the abnormal state of the gut microbiota, as well as chronic inflammation in chronic liver diseases, but short-term probiotic treatment still has no effect on adjusting intestinal permeability or liver function $[47,48]$. With the in-depth study of gut microbiota, someone suggested that fecal microbiota transplantation (FMT) will be a promising treatment for chronic HBV infection. FMT refers to the infusion of a faecal suspension from healthy people into the intestinal tract of patients to cure a specific disease [32]. More and more researches showed that FMT has been used to treat Clostridium difficile infection, IBD, IBS, and various liver diseases with some effects [49]. A 2015 case report described that FMT can reduce blood ammonia, increase cognitive abilities, and improve hepatic encephalopathy for a 57-year-old male with cirrhosis secondary to both alcohol and the hepatitis $\mathrm{C}$ virus [50]. A report on $\mathrm{HBeAg}$-positive CHB therapy in patients with ongoing ETV/ TDF therapy showed that FMT can induce HBeAg clearance in a significant proportion of cases who have persistent positive $\mathrm{HBeAg}$ even after long-term antiviral treatment. Although only 5 patients participated in the FMT group, the statistics varied significantly $(P=0.0001)$ and these dramatic results were particularly encouraging for patients with $\mathrm{HBeAg}$-positive $\mathrm{CHB}$ who could not cease oral antiviral therapy [51]. Therefore, FMT may be as a potential immunomodulator in improving the intestinal microenvironment and alleviating the damage to the liver caused by harmful intestinal bacteria [32]. However, there are also associated ethical, legal, and social problems; we should establish a balance of scientific research, health, and marketing regarding the study of the intestinal flora [52]. And the data on the field of FMT treatment related to HBV-related diseases is still limited. So there will be a lot of work to do on FMT and chronic HBV infection for us in the future [32].

\section{Conflicts of Interest}

The authors declare that they have no conflicts of interest.

\section{Acknowledgments}

This work was supported by the National Science Foundation of China (no. 81500430 and no. U1304802), the Science and Technology Planning Project of Henan Province (no. 182102310567 and no. 182102310544), the Science and Technology Tackling Project of Henan Province (no. 201702136), and the Key Project of Science and Technology Research of Education Department of Henan Province (no. 17A320019).

\section{References}

[1] World Health Organization, Global Hepatitis Report 2017, WHO, 2017, http://www.who.int/hepatitis.

[2] S. Beckebaum, M. Malagó, O. Dirsch et al., "Efficacy of combined lamivudine and adefovir dipivoxil treatment for severe HBV graft reinfection after living donor liver transplantation," Clinical Transplantation, vol. 17, no. 6, pp. 554-559, 2003.

[3] C. Castelnau, F. le Gal, M. P. Ripault et al., "Efficacy of peginterferon alpha-2b in chronic hepatitis delta: relevance of quantitative RT-PCR for follow-up," Hepatology, vol. 44, no. 3, pp. 728-735, 2006.

[4] P. Hartmann, W. C. Chen, and B. Schnabl, "The intestinal microbiome and the leaky gut as therapeutic targets in alcoholic liver disease," Frontiers in Physiology, vol. 3, p. 402, 2012.

[5] R. Lin, L. Zhou, J. Zhang, and B. Wang, "Abnormal intestinal permeability and microbiota in patients with autoimmune hepatitis," International Journal of Clinical and Experimental Pathology, vol. 8, no. 5, pp. 5153-5160, 2015.

[6] M. Bhat, B. M. Arendt, V. Bhat, E. L. Renner, A. Humar, and J. P. Allard, "Implication of the intestinal microbiome in complications of cirrhosis," World Journal of Hepatology, vol. 8, no. 27, pp. 1128-1136, 2016.

[7] M. Grąt, K. M. Wronka, M. Krasnodębski et al., "Profile of gut microbiota associated with the presence of hepatocellular cancer in patients with liver cirrhosis," Transplantation Proceedings, vol. 48, no. 5, pp. 1687-1691, 2016.

[8] D. E. Fouts, M. Torralba, K. E. Nelson, D. A. Brenner, and B. Schnabl, "Bacterial translocation and changes in the intestinal microbiome in mouse models of liver disease," Journal of Hepatology, vol. 56, no. 6, pp. 1283-1292, 2012.

[9] H. H. Chou, W. H. Chien, L. L. Wu et al., "Age-related immune clearance of hepatitis $\mathrm{B}$ virus infection requires the establishment of gut microbiota," Proceedings of the National Academy of Sciences, vol. 112, no. 7, pp. 2175-2180, 2015.

[10] A. J. Prendergast, P. Klenerman, and P. J. R. Goulder, "The impact of differential antiviral immunity in children and adults," Nature Reviews Immunology, vol. 12, no. 9, pp. 636648, 2012.

[11] K. N. Tsai, C. F. Kuo, and J. H. J. Ou, "Mechanisms of hepatitis B virus persistence," Trends in Microbiology, vol. 26, no. 1, pp. 33-42, 2018. 
[12] D. Xu, Y. Huang, and J. Wang, "Gut microbiota modulate the immune effect against hepatitis B virus infection," European Journal of Clinical Microbiology \& Infectious Diseases, vol. 34, no. 11, pp. 2139-2147, 2015.

[13] A. Busca and A. Kumar, "Innate immune responses in hepatitis B virus (HBV) infection," Virology Journal, vol. 11, no. 1, p. 22, 2014.

[14] E. Zhang and M. Lu, "Toll-like receptor (TLR)-mediated innate immune responses in the control of hepatitis B virus (HBV) infection," Medical Microbiology and Immunology, vol. 204, no. 1, pp. 11-20, 2015.

[15] Z. Ma, E. Zhang, D. Yang, and M. Lu, "Contribution of Toll-like receptors to the control of hepatitis B virus infection by initiating antiviral innate responses and promoting specific adaptive immune responses," Cellular \& Molecular Immunology, vol. 12, no. 3, pp. 273-282, 2015.

[16] K. Visvanathan, N. A. Skinner, A. J. V. Thompson et al., "Regulation of Toll-like receptor-2 expression in chronic hepatitis B by the precore protein," Hepatology, vol. 45, no. 1, pp. 102-110, 2007.

[17] D. Das, I. Sengupta, N. Sarkar et al., "Anti-hepatitis B virus (HBV) response of imiquimod based toll like receptor 7 ligand in hbv-positive human hepatocelluar carcinoma cell line," BMC Infectious Diseases, vol. 17, no. 1, p. 76, 2017.

[18] I. Sekirov, S. L. Russell, L. C. M. Antunes, and B. B. Finlay, "Gut microbiota in health and disease," Physiological Reviews, vol. 90, no. 3, pp. 859-904, 2010.

[19] H. Fukui, "Gut microbiota and host reaction in liver diseases," Microorganisms, vol. 3, no. 4, pp. 759-791, 2015.

[20] K. Korpela, A. Salonen, L. J. Virta et al., "Intestinal microbiome is related to lifetime antibiotic use in Finnish pre-school children," Nature Communications, vol. 7, article 10410, 2016.

[21] R. Sender, S. Fuchs, and R. Milo, "Revised estimates for the number of human and bacteria cells in the body," PLoS Biology, vol. 14, no. 8, article e1002533, 2016.

[22] K. Atarashi and K. Honda, "Microbiota in autoimmunity and tolerance," Current Opinion in Immunology, vol. 23, no. 6, pp. 761-768, 2011.

[23] M. Rajilić-Stojanović, D. M. Jonkers, A. Salonen et al., "Intestinal microbiota and diet in IBS: causes, consequences, or epiphenomena?," The American Journal of Gastroenterology, vol. 110, no. 2, pp. 278-287, 2015.

[24] S. Michail, M. Durbin, D. Turner et al., "Alterations in the gut microbiome of children with severe ulcerative colitis," Inflammatory Bowel Diseases, vol. 18, no. 10, pp. 1799-1808, 2012.

[25] L.-M. Haag and B. Siegmund, "Intestinal microbiota and the innate immune system - a crosstalk in Crohn's disease pathogenesis," Frontiers in Immunology, vol. 6, p. 489, 2015.

[26] J. D. Forbes, G. van Domselaar, and C. N. Bernstein, "The gut microbiota in immune-mediated inflammatory diseases," Frontiers in Microbiology, vol. 7, article 1081, 2016.

[27] F. R. Ponziani, S. Bhoori, C. Castelli et al., "Hepatocellular carcinoma is associated with gut microbiota profile and inflammation in non-alcoholic fatty liver disease," Hepatology, 2018.

[28] H. C. Xing, L. J. Li, K. J. Xu et al., "Protective role of supplement with foreign Bifidobacterium and Lactobacillus in experimental hepatic ischemia-reperfusion injury," Journal of Gastroenterology and Hepatology, vol. 21, no. 4, pp. 647656, 2006.
[29] H. Lu, Z. Wu, W. Xu, J. Yang, Y. Chen, and L. Li, "Intestinal microbiota was assessed in cirrhotic patients with hepatitis B virus infection. Intestinal microbiota of $\mathrm{HBV}$ cirrhotic patients," Microbial Ecology, vol. 61, no. 3, pp. 693-703, 2011.

[30] D. W. Jun, K. T. Kim, O. Y. Lee et al., "Association between small intestinal bacterial overgrowth and peripheral bacterial DNA in cirrhotic patients," Digestive Diseases and Sciences, vol. 55, no. 5, pp. 1465-1471, 2010.

[31] J. Wang, Y. Wang, X. Zhang et al., "Gut microbial dysbiosis is associated with altered hepatic functions and serum metabolites in chronic hepatitis B patients," Frontiers in Microbiology, vol. 8, p. 2222, 2017.

[32] Y. Kang and Y. Cai, "Gut microbiota and hepatitis-B-virusinduced chronic liver disease: implications for faecal microbiota transplantation therapy," Journal of Hospital Infection, vol. 96, no. 4, pp. 342-348, 2017.

[33] P. Brun, I. Castagliuolo, V. D. Leo et al., "Increased intestinal permeability in obese mice: new evidence in the pathogenesis of nonalcoholic steatohepatitis," American Journal of Physiology-Gastrointestinal and Liver Physiology, vol. 292, no. 2, pp. G518-G525, 2007.

[34] T. H. Frazier, J. K. DiBaise, and C. J. McClain, "Gut microbiota, intestinal permeability, obesity-induced inflammation, and liver injury," Journal of Parenteral and Enteral Nutrition, vol. 35, 5_Supplement, pp. 14s-20s, 2011.

[35] E. Seki and B. Schnabl, "Role of innate immunity and the microbiota in liver fibrosis: crosstalk between the liver and gut," The Journal of Physiology, vol. 590, no. 3, pp. 447-458, 2012.

[36] M. Bermudez-Brito, S. Muñoz-Quezada, C. Gomez-Llorente et al., "Cell-free culture supernatant of Bifidobacterium breve CNCM I-4035 decreases pro-inflammatory cytokines in human dendritic cells challenged with Salmonella typhi through TLR activation," PLoS One, vol. 8, no. 3, article e59370, 2013.

[37] A. Borrelli, P. Bonelli, F. M. Tuccillo et al., "Role of gut microbiota and oxidative stress in the progression of non-alcoholic fatty liver disease to hepatocarcinoma: current and innovative therapeutic approaches," Redox Biology, vol. 15, pp. 467-479, 2018.

[38] Y. H. Paik, R. F. Schwabe, R. Bataller, M. P. Russo, C. Jobin, and D. A. Brenner, "Toll-like. Receptor 4 mediates inflammatory signaling by bacterial lipopolysaccharide in human hepatic stellate cells," Hepatology, vol. 37, no. 5, pp. 10431055, 2003.

[39] L. Lin and J. Zhang, "Role of intestinal microbiota and metabolites on gut homeostasis and human diseases," BMC Immunology, vol. 18, no. 1, p. 2, 2017.

[40] K. Gao, L. Liu, and H. Wang, "Advances in immunomodulation of microbial unmethylated CpG DNA on animal intestinal tract-a review," Acta Microbiologica Sinica, vol. 55, no. 5, pp. 543-550, 2015.

[41] N. Cools, A. Petrizzo, E. Smits et al., "Dendritic cells in the pathogenesis and treatment of human diseases: a Janus Bifrons?," Immunotherapy, vol. 3, no. 10, pp. 1203-1222, 2011.

[42] M. U. Cinar, M. A. Islam, M. Pröll et al., "Evaluation of suitable reference genes for gene expression studies in porcine PBMCs in response to LPS and LTA," BMC Research Notes, vol. 6, no. 1, p. 56, 2013.

[43] B. Lemaitre and S. E. Girardin, "Translation inhibition and metabolic stress pathways in the host response to bacterial 
pathogens," Nature Reviews Microbiology, vol. 11, no. 6, pp. 365-369, 2013.

[44] T. Kawasaki, T. Kawai, and S. Akira, "Recognition of nucleic acids by pattern-recognition receptors and its relevance in autoimmunity," Immunological Reviews, vol. 243, no. 1, pp. 61-73, 2011.

[45] A. Ignacio, C. I. Morales, N. O. S. Câmara, and R. R. Almeida, "Innate sensing of the gut microbiota: modulation of inflammatory and autoimmune diseases," Frontiers in Immunology, vol. 7, p. 54, 2016.

[46] R. Codella, L. Luzi, and I. Terruzzi, "Exercise has the guts: how physical activity may positively modulate gut microbiota in chronic and immune-based diseases," Digestive and Liver Disease, vol. 50, no. 4, pp. 331-341, 2018.

[47] Y. Liang, S. Liang, Y. Zhang et al., "Oral administration of compound probiotics ameliorates HFD-Induced gut microbe dysbiosis and chronic metabolic inflammation via the $G$ protein-coupled receptor 43 in non-alcoholic fatty liver disease rats," Probiotics and Antimicrobial Proteins, pp. 1-11, 2018.

[48] D. S. Kwak, D. W. Jun, J. G. Seo et al., "Short-term probiotic therapy alleviates small intestinal bacterial overgrowth, but does not improve intestinal permeability in chronic liver disease," European Journal of Gastroenterology \& Hepatology, vol. 26, no. 12, pp. 1353-1359, 2014.

[49] R. D. Heath, C. Cockerell, R. Mankoo, J. A. Ibdah, and V. Tahan, "Fecal microbiota transplantation and its potential therapeutic uses in gastrointestinal disorders," Northern Clinics of Istanbul, vol. 5, no. 1, pp. 79-88, 2018.

[50] T.-C. D. Shen, L. Albenberg, K. Bittinger et al., "Engineering the gut microbiota to treat hyperammonemia," The Journal of Clinical Investigation, vol. 125, no. 7, pp. 2841-2850, 2015.

[51] Y. D. Ren, Z. S. Ye, L. Z. Yang et al., "Fecal microbiota transplantation induces hepatitis B virus e-antigen ( $\mathrm{HBeAg}$ ) clearance in patients with positive $\mathrm{HBeAg}$ after long-term antiviral therapy," Hepatology, vol. 65, no. 5, pp. 1765-1768, 2017.

[52] Y. Ma, H. Chen, C. Lan, and J. Ren, "Help, hope and hype: ethical considerations of human microbiome research and applications," Protein \& Cell, vol. 9, no. 5, pp. 404-415, 2018. 


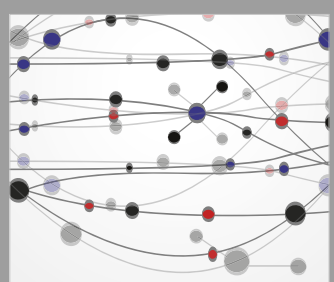

The Scientific World Journal
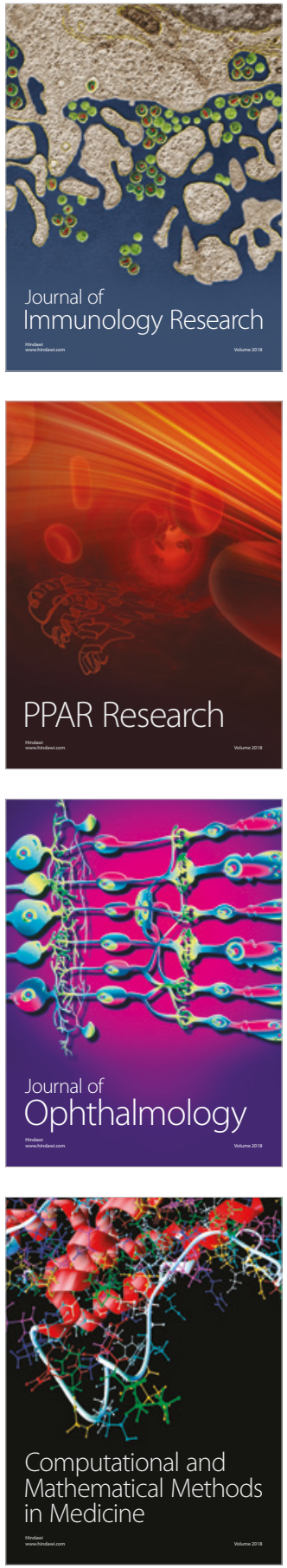

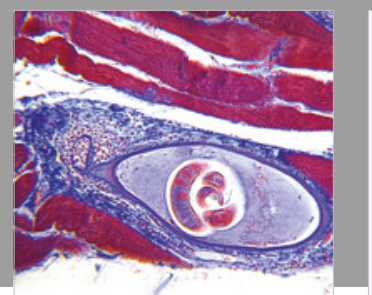

Gastroenterology Research and Practice

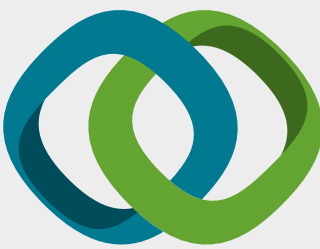

\section{Hindawi}

Submit your manuscripts at

www.hindawi.com
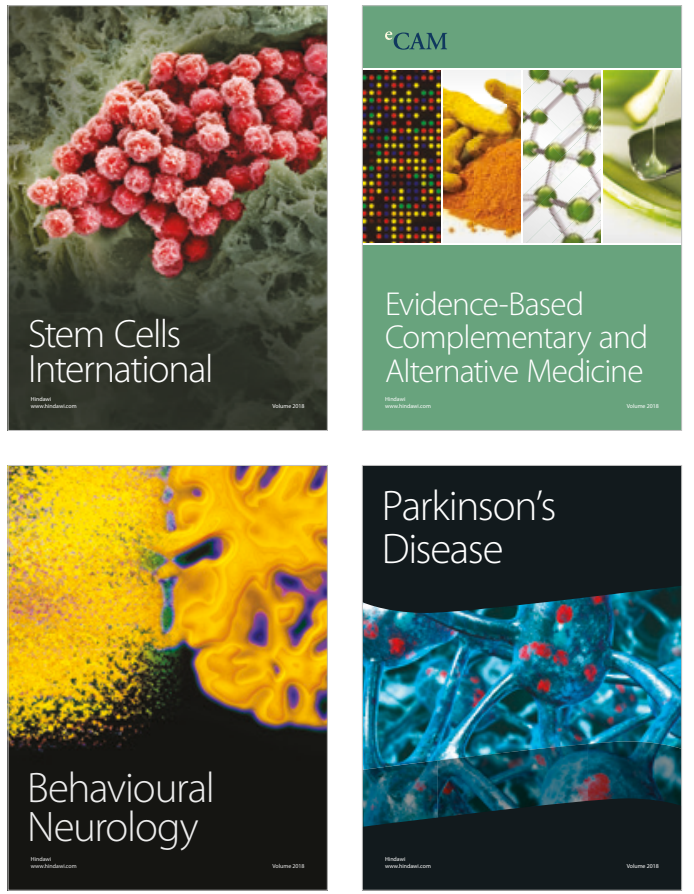

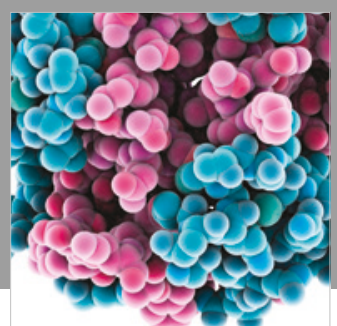

ournal of

Diabetes Research

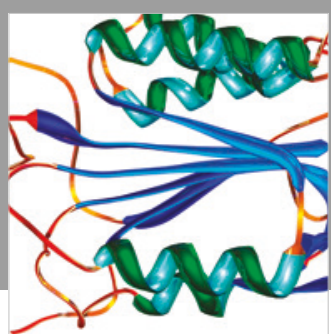

Disease Markers
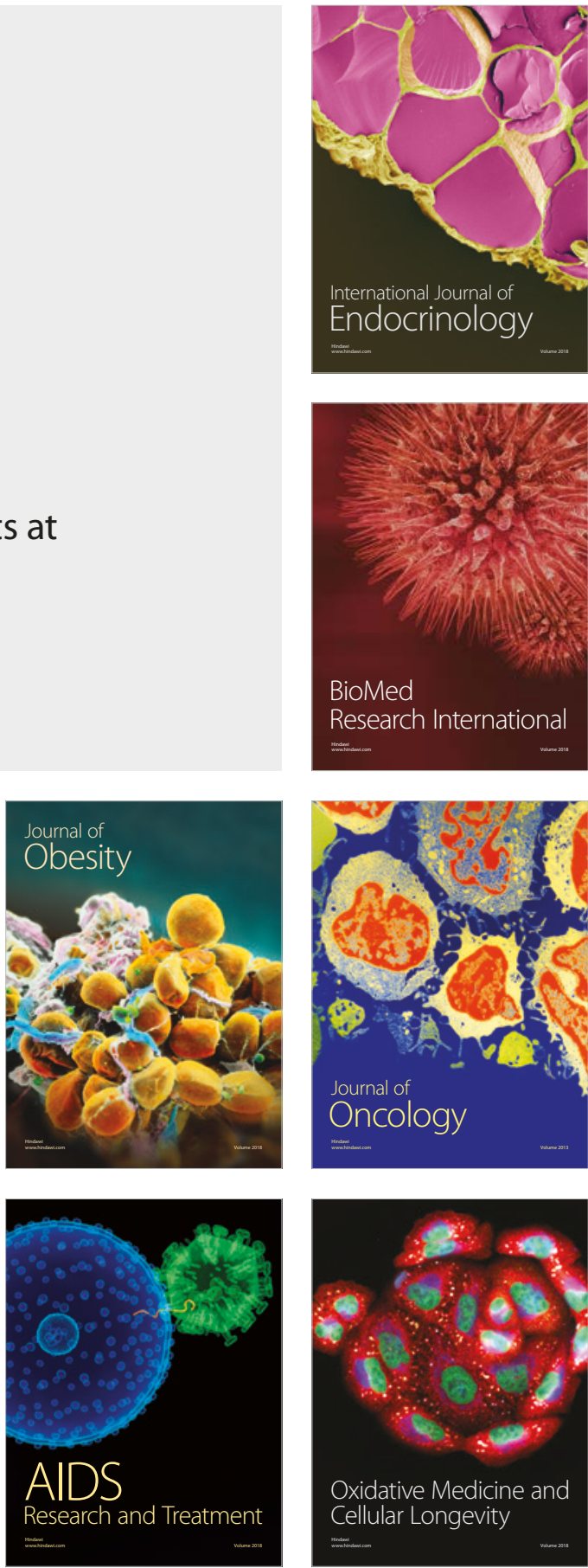